\title{
Perspective View on Sorption Thermodynamics: Basic Dye Uptake on Southern Nigerian Clay
}

\author{
Daniel C. Emeniru \\ John Neminebor \\ Department of Petroleum Engineering and Geoscience technology, \\ Federal Polytechnic, Ekowe, Bayelsa state, Nigeria.

\section{Justin Ikirigo} \\ Department of Science Laboratory Technology, Federal Polytechnic, \\ Ekowe, Bayelsa state, Nigeria.

\section{Franklin Sogbara} \\ Department of Mechanical Engineering Technology, Federal Polytechnic, \\ Ekowe, Bayelsa state, Nigeria.
}

doi: 10.19044/esj.2017.v13n18p355 URL:http://dx.doi.org/10.19044/esj.2017.v13n18p355

Abstract

Macroscopic phenomenon like adsorption has a mechanistic tie typical of thermodynamics and its principles. This work examined the thermodynamic parameters for methylene blue (MB) uptake onto modified Ekowe clay (EC). The purified clay was calcined for $4 \mathrm{hrs}$. at $750^{\circ} \mathrm{C}$ to obtain Natural Ekowe Clay (NEC). The purified clay was activated $\left(1.6 \mathrm{M} \mathrm{H}_{2} \mathrm{SO}_{4}\right.$ (aq)) and calcined for $4 \mathrm{hrs}$. at $750^{\circ} \mathrm{C}$ obtaining Activated Ekowe Clay (AEC). Thermodynamic study applied the equilibrium data in determining the activation and heats of adsorption parameters. The concave Eyring plot suggests more than one rate-limiting steps coexisting in the sorption. For temperatures: 25,30 and $40^{\circ} \mathrm{C}$, activation energies $(\mathbb{E})$ for NEC and AEC lie between 2 - 29kJ/mol inferring physisorption. Negative activation enthalpies $\left(\Delta \mathrm{H}^{*}\right)$ values confirm exothermic activations. The less negative $\Delta \mathrm{H}^{*}$ values, in compliance with the significant $\mathrm{k}_{2}$ values varying inversely with temperature, suggests high sorption rate. The negative activation entropy explained an associative uptake and the less negative values are attributive to a physical uptake. Negative free activation enthalpy, $\Delta \mathrm{G}^{*}$ indicated that uptake on the modified EC is spontaneous. High negativity of $\Delta \mathrm{G}^{*}$ values suggest strong physic-sorption bond. The negative $\Delta \mathrm{H}^{*}, \Delta \mathrm{S}^{*}$ and $\Delta \mathrm{G}^{*}$ values characterize the physisorption of MB onto modified EC. Values of the isoexcess heats $\left(\boldsymbol{q}_{\text {isox }}\right)$ obtained: $2.67 \mathrm{~kJ} / \mathrm{mol}$. (NEC) and $2.47 \mathrm{~kJ} / \mathrm{mol}$. (AEC) agreed with the value range of $<80 \mathrm{~kJ} / \mathrm{mol}$. typical to physic-sorption. This work opines that sorption of $\mathrm{MB}$ onto modified $\mathrm{EC}$ is a spontaneous 
exothermic multilayer phenomenon that progresses heterogeneously with continuous decrease in sorption potential and fall in isosteric heat.

Keywords: Adsorption, thermodynamics, activation, Iso-heat

\section{Note}

Sorbtive is dye in the bulk solution; Sorbate is the adsorbed dye in the sorbent surface, Activation enthalpy is same as enthalpy of activation and Sorption was used for adsorption.

\section{Introduction}

Adsorption has been reported to be one of the most powerful treatment processes for the removal of dyes from wastewater; hence the need to enhance the mechanisms, procedures and materials involved in the adsorption technologies so as to improve effectiveness.

Studies have proven clay adsorbent recyclable (regeneration), abundant (availability), cheap (affordability) and user/application friendly (Emeniru et al., 2015). The sorption potency of clay can be traced to the clay minerals (hydrous aluminum silicates), the large interlayer space capable of holding significant amounts of absorbable substances, and its characteristic large surface area. Unlike acid activation that has gained much popularity in the adsorption research space, literature has reported sparingly on the application of calcinations of either raw or acid activated clay.

Dye has covert and accumulative adversities on mac2hineries and living organisms. Its complex structure, synthetic origin and recalcitrant behavior to remediation in the soil and water have defied most materials and methods for its environmental remediation. Therefore, the clay from Ekowe - an area in the core of the Niger Delta region of southern Nigeria, was considered to study the sorption thermodynamics of Basic dye uptake.

Adsorption thermodynamics expresses as a function of surface concentration, chemical potential and composition, temperature and other state variables, the energy requirement for particulate surface phenomenon; defining the chemical state and kinetic of the system, the capacity of the sorbent, maximum absorbable amount, effective sorption sites, and surface affinity and bond strength. Relatively, it sets to provide answers to: how many particles can be adsorbed by a given amount of material, with surface concentration $\mathrm{C}_{\mathrm{ad}}$ (moles $/ \mathrm{m}^{2}$ ) being the thermodynamic quantity of interest; how does this amount depend on the state variables (temperature, pressure and concentration), i.e. how can one control the sorbate concentration by an appropriate choice of $\mathrm{T}, \mathrm{P}$ and $\mathrm{C}$; how can the stationary (equilibrium) and maximum uptake be determined; where are the adsorbed particles located at, 
on, and in the surface, respectively; and how much energy is required to remove these particles from the surface - how strong are the sorptive bonds to the surface? Thermodynamics has the remarkable ability to connect seemingly unrelated properties (Myers, 2004): the temperature coefficient of sorption is directly proportional to the heat of immersion of the solid sorbent (Myers, 2004). The most important application of thermodynamics to adsorption is the calculation of phase equilibrium between a sorptive and a solid sorbent (Myers, 2004), and the equilibrium sorption isotherm, measurable experimentally or calculated theoretically, which gives the uptake in the pores as a function of the sorptive bulk concentration. At constant process temperature and pressure, before the establishment of significant intra-particle diffusion and sorbate adhesion on sorbent surfaces, Gibbs postulate noted equilibration of sorptive chemical/transfer potential between the solid sorbent vicinity and the bulk fluid leading to constant mass concentration in the system.

At any instant in sorption process, the amount of the sorptive at the vicinity of the sorbent cannot be estimated experimentally owing that it is not practicable to generate the density profile of the sorptive located within the sorbent surface vicinity, though, it can be expressed theoretically and referred as the absolute uptake; relatively, what can and have been estimated (instantaneously or at equilibrium) is the excess uptake which represent the amount of sorbate on the sorbent surface/vicinity in excess of the amount that is present at the instantaneous/equilibrium density of the bulk. The absolute uptake $\left(q_{a}\right)$ and excess uptake $\left(q_{e x}\right)$ are related by

$$
q_{a}=q_{e x}+V_{a d s} \rho_{i}(T)
$$

The volume of the vicinity around sorbent surface where the impracticable density profile resides $\left(\mathrm{V}_{\mathrm{ads}}\right)$ is fundamentally unknown, and $\rho_{i}$ is the instantaneous concentration of the sorptive at experimental temperature. These points to the fact that the true thermodynamics of a sorption process revolves around the excess amount adsorbed. Therefore, the simplest approach to thermodynamic calculations using experimental isotherm data is to proceed with the measured $q_{e x}$. That is to say, in sorption experiments, standard volumetric measurements are known to measure s8urface excess amount, $q_{e}$. It follows that at constant temperature in the early stage of a batch solid/liquid sorption medium where the sorptive pressure effect is insignificant (Pressure approx. zero) the instantaneous effective mass concentration on the sorbent surface vicinity is theoretically zero $\left(\rho_{i}=\frac{P}{R T}=0\right)$ implying that the excess uptake is a limit of the absolute uptake as the sorptive pressure on the system tends to zero, thus expressed as: 


$$
\lim _{P \rightarrow 0}\left(q_{a}\right)=\lim _{P \rightarrow 0}\left(q_{e x}+\frac{P}{R T} V_{a d s}\right)=q_{e x}+0=q_{e x}
$$

Hence it can be inferred that at fixed system temperature and pressure for adsorption on porous and non-porous solids, the sorptive effective mass concentration, $\rho\left(\mathrm{C}_{\mathrm{a}}\right)$ relative to the excess uptake at $\mathrm{t} \approx 0$, is zero. The mass Concentration at the solid surface and beyond is an unknown function of the distance from the surface, and at a certain distance from the surface, the density is equal to the bulk density.

The concept and application of thermodynamics and kinetics are highly synergetic; that is, thermodynamically, the Langmuir isotherm theory and parameters as often considered in the Van Hoff equation, has a simple kinetic derivation that depends on the assumption of dynamic equilibrium.

\section{Thermodynamics Activation}

Sorption proceed by the formation of activation complex between the sportive and the sorbent which progresses by dissociation to form new product as in catalysis (chemis-orption) or the complex is held intact by the weak intermolecular force hence the sorptive can desorb as in physicsorption. Considering that adsorption is a surface phenomenon (a physical process) often characterized by desorption and likened to the formation of activation complex in chemical reaction, the adsorbed phase is the transition state when there is thermodynamic equilibrium between the adsorbed and the bulk phases driven by the proportionality of adsorption rate and the adsorbed phase concentration, $\mathrm{C}_{\mathrm{ad}}$ (or the amount adsorbed). In adsorption, as relates chemical and environmental engineering, energy (binding, rotational, vibration and/or translational) must be acquired or overcome by sorptives to interact with sorbent surfaces (physically or chemically) even when the process is spontaneous. Eyring modified the Arrhenius equation to accommodate the study of solution reactions and mixed phase reactions where the simple collision model is not very helpful, though it is a theoretical construct, based on transition state theory. Adsorption activation energy, $\mathbb{E}$ $\left(\mathrm{kJ} \mathrm{mol}{ }^{-1}\right)$ can be determined from experimental measurements of the adsorption rate constant at different temperatures according to Arrhenius postulate and the Eyring equation. Based on statistical and quantum mechanism, the Eyring equation (3) takes into account the standard vibrational partition function, equivalent to the frequency factor of the Arrhenius equation and given as $\frac{k_{B} T}{h}$ :

$$
k_{a}=\frac{k_{B} T}{h} \mathrm{\kappa}
$$


Where the Quasi-thermodynamic equilibrium constant, $\kappa=e^{-\frac{\Delta G^{*}}{R T}}, k_{B}$ is Boltzman constant $\left(1.3807 \times 10^{-23} \mathrm{~J} / \mathrm{K}\right)$, and $h$ is Plank constant $\left(6.6261 \times 10^{-34} \mathrm{Js}\right), \quad k_{a}$ is sorption rate constant. The rate constant can be obtained by inputting the experimental equilibrium data into the various kinetic models and using that of the model with lowest Standard error and normalized standard deviation. Contrary to the first-order, the second order model. The second order model predicts the sorption behavior over a whole uptake time (Ho, 2006) and uses only the experimental uptakes; is more logical compared to the first order that uses estimated equilibrium uptake. The second-order kinetic model is expressed as (Ho, 1995):

$$
\frac{d q_{t}}{d t}=k_{2}\left(q_{e}-q_{t}\right)^{2}
$$

Integration and linearized at boundary conditions of $t=0$ to $t=\mathrm{t}$ and $q=0$ to $q=q_{t}$

$\left(\frac{t}{q_{t}}\right)=\left(\frac{1}{k_{2} q_{e}^{2}}\right)+\left(\frac{1}{q_{e}}\right)$

The plot of $t / q_{t}$ against $t$ gives a linear where the rate constant $\left(\mathrm{k}_{\mathrm{a}}\right)$ and equilibrium update $\left(q_{\mathrm{e}}\right)$ could be determined from the intercept and slope of the plot, respectively.

Therefore,

$$
k_{a}=\frac{k_{B} T}{h} e^{-\left(\frac{\Delta \mathrm{H}^{*}-T \Delta \Delta^{*}}{R T}\right)}
$$

Taking natural log $(\ln )$ we have

$$
\ln \left(\frac{k_{a}}{T}\right)=\ln \left(\frac{k_{B}}{h}\right)+\frac{\Delta S^{*}}{R}-\frac{\Delta H^{*}}{R}\left(\frac{1}{T}\right)
$$

Where $\mathrm{R}$ is universal constant $(8.314 \mathrm{~J} / \mathrm{mol} . \mathrm{K}), \mathrm{T}=$ absolute temperature $(\mathrm{K})$; Eyring plot of $\ln \left(\frac{k_{a}}{T}\right) v s\left(\frac{1}{T}\right)$ gives a slope of $\frac{\Delta H^{*}}{R}$ and intercept of $\ln \left(\frac{k_{B}}{h}\right)+\frac{\Delta S^{*}}{R}$ from which enthalpy $\left(\Delta \mathrm{H}^{*}\right)$ and entropy $\left(\Delta \mathrm{S}^{*}\right)$ of activation can be estimated as well as the activation free energy $\left(\Delta G^{*}\right)$ : equation (8). Considering the equilibrium between the adsorbed and bulk phases expressed as

$$
C_{a e}=\kappa C_{e}
$$

The Quasi-equilibrium constant, $\kappa$ can be estimated numerically and the free energy $\left(\Delta \mathrm{G}^{*}\right)$ calculated from the expression:

$\Delta G^{*}=-R T \ln \kappa$ 
However, from $\Delta \mathrm{H}^{*}$ and $\Delta \mathrm{S}^{*}$ obtained (graphically) from the Eyring plot, the free activation energy, $\Delta \mathrm{G}^{*}$ can be estimated from the popular thermodynamic relationship:

$$
\Delta G^{*}=\Delta H^{*}+T \Delta S^{*}
$$

The Activation free energy, $\Delta \mathrm{G}^{*}$ which indicate the extent and spontaneity of the adsorption explains the following; $\Delta \mathrm{G}^{*}<0$ implies a spontaneous adsorption, $\Delta \mathrm{G}^{*}=0$ implies an equilibrium process where no net change is noticed and $\Delta \mathrm{G}^{*}>0$ depicts the adsorption is not spontaneous.

A precise determination of the activation parameters requires sorption kinetic runs at varied experimental temperatures of at least $5^{\circ} \mathrm{C}$ intervals to determine the applicable sorption rate constant, $k_{a}$ and though the estimation of these parameters must be performed accurately, it should not pretend an excessive accuracy. A plot showing concave curve towards the temperature axis explains the presence of at least two different sorption rate-limiting steps. A convex curve may imply that $\Delta \mathrm{H}^{*}$, decreases with increasing temperature.

Generally, $\Delta \mathrm{G}^{*}$ values are often positive at all temperatures suggesting that sorption reactions require some external energy whether inherent or induced (saha and Chowdhury, 2010). Processes that exhibit negative $\Delta \mathrm{H}^{*}$ are exothermic in nature and proceed at low temperatures, however positive activation energies $\left(+\Delta \mathrm{H}^{*}\right)$ typical of endothermic sorption have most frequently been reported for dye stuff: Alkan et al., 2008 reported $19.25 \mathrm{~kJ} / \mathrm{mol}$ for Maxilon Blue $5 \mathrm{G}$ onto Sepiolite; $24.24 \mathrm{~kJ} / \mathrm{mol}$ have been recorded for Methylene Blue onto Modified wheat straw (Han et al., 2010); Malachite Green onto Sea shell powder gave $15.71 \mathrm{~kJ} / \mathrm{mol}$ (Chowdhury and Saha, 2010); Aksakal and Ucun, 2010 observed 8.904kJ/mol for uptake of Reactive Red 195 on to Pinus sylvestris L.Exothermicity of activation is often concomitant to negativity of the process enthalpy, $\Delta \mathrm{H}$ (exothermic uptake process) except often with chemisorption where new endothermically driven product is expected. The dynamics of chemisorption is known to be dependent on the sorption energy induced by the bond energy (heat of dissociation) of the species/sorptive as a function of their distance to the sorbent. Though the activation energy, $\mathbb{E}$ and the activation enthalpy, $\Delta \mathrm{H}^{*}$ are often reported interchangebly in the literatures, they are related as:

$$
E=\Delta H^{*}+R T
$$

Where low values of $\mathbb{E}$ and $\Delta \mathrm{H}^{*}(<20 \mathrm{~kJ} / \mathrm{mol})$ depicts a fast adsorption rate while high values $\left(20<\mathbb{E} / \Delta \mathrm{H}^{*}<150 \mathrm{~kJ} / \mathrm{mol}\right)$ implies slow rate.

In adsorption, adsorbed species transitions from their free state (with three degrees of translational freedom) to the adsorbed film (with two 
degrees of translational freedom) and therefore loses translational entropy. The activation entropy $\left(\Delta \mathrm{S}^{*}\right)$ indicate whether sorption is associative (molecular, $-\Delta S^{*}$ ): leaving the sorbing molecule intact with no significant change occured in the internal structure of the sorbent, an attribute of physisorption; or dissociative (atomic, $+\Delta \mathrm{S}^{*}$ ): cleavage of the sorbing molecule either in a homolytic or heterolytic manner, characteristic of chemisorption. In physisorption, the activated complex in the transition state (when sorption/reaction rate is proportional to the concentration of the activated complex) has a more ordered structure than the sorptives in the bulk. The translational, rotational and/or vibrational excitement at the transition state compared to the bulk became negligible and ineffective. This sorption is favoured at low temperature. When the complex is highly disordered compared to the sorptive in the bulk, and the translational, rotational and vibrational mobility of the sorbate in the transition state (complex) is enhanced, Chemisorption, favoured at high temperature characterize the sorption.

\section{Heat of Adsorption}

In surface sorption process, the value of the sorption heat varies with the sorbent surface coverage indicating that the energy requirement at the sorbent surface varies as sorption progresses. As a result, the sorbent surface during uptake is energetically heterogeneous, giving the heat an implicit character of giving information on the degree of sorbent heterogeneity. For homogeneous surfaces, as sorption proceeds, the heat is constant as the surface coverage varies. Sorption heat is usually high at very low coverage and decreases steadily with an increase in uptake, q (Saha and Chowdhury, 2010). At the onset or early stage of an uptake process, the sorbent surface sites are highly active possessing high sorption potential. As sorption progresses, the sorbent surface monolayer coverage increases with decreasing potential and increasing heat relative to the uptake onset; multilayer sorption, characterized by sorbate-sorbate interaction and further increase in uptake (q), sets in with further decrease in sorption potential and fall in the heat. Therefore, high sorption potential is characteristic of low uptake, $\mathrm{q}$ and high heat, $\mathrm{q}_{\mathrm{i}}$.

\section{Isosteric Heat}

The heat consideration for the sorption system centers on the differential energy of sorption given as the change in internal energy of the entire system upon the addition of an infinitesimal surface excess amount dq. Hence, on the stance of volumetric analysis, the differential Gibbs energy change of a sorption system can be expressed as: 


$$
\begin{aligned}
& d G=\left(\frac{\partial G}{\partial P}\right)_{T, A} d P=\left(\frac{\partial G}{\partial T}\right)_{P, A} d T=\left(\frac{\partial G}{\partial A}\right)_{P, T} d A=\left(\frac{\partial G}{\partial n}\right)_{P, T, A} d n \\
& d G=V d P-S d T+\sigma d A+\mu d n
\end{aligned}
$$

With $\mathrm{P}=$ pressure, $\mathrm{T}=$ temperature, $\mathrm{A}=$ surface area, $\sigma=$ surface potential (entirely equivalent to the surface energy which is the driving force for all surface phenomena.), $\mathrm{V}=$ system volume, $\mu=$ chemical potential and $\mathrm{S}=$ entropy. There is no limit to the number of heats of sorption which can be expressed based on the process thermodynamic functions of Pressure, Temperature, surface concentration, and surface coverage kept constant. As here considered, constant Temperature and pressure, isothermal and isosteric conditions apply, thus the differential enthalpy of adsorption $\left(d h_{T, C_{a}}\right)$ can be estimated.

The most relevant thermodynamic variable to describe the heat effects during sorption process is the isosteric heat (Saha and Chowdhury, 2010) characteristic of constant surface concentration. At equilibrium (dynamic), when the fluxes of adsorbing sorptive and desorbing sorbate are equal, constant sorptive concentration within the surface excess phase (surface concentration) is maintained and the Gibbs energy change became zero leading to "persisting" equilibrium where the chemical potential of the adsorbed phase $\left(\mu^{a}\right)$ being persistently equals that of the bulk phase $\left(\mu^{b l k}\right)$ : that is $\mu=\left(\frac{\partial G}{\partial n}\right)_{P, T, A}$ and $\mu^{a}=\mu^{b l k}$. Therefore, assuming that the thermodynamic properties of the solid surface remain unchanged upon adsorption, we have:

$$
\mu^{a}=V_{a} d P-S_{a} d T=\mu^{b l k}=V_{b} d P-S_{b} d T
$$

Recalling that sorption binding energies can be estimated from the temperature dependence of adsorption/desorption equilibrium concentration based on partial molar quantitythe above expression becomes the popular Clausius-Clapeyron equation given as:

$$
\frac{s_{b l k}-s_{a}}{v_{b l k}-v_{a}}=\left(\frac{d P}{d T}\right)_{C_{a}}=\frac{\Delta s}{\Delta v}
$$

Noting that $\mathrm{S}, \mathrm{V}$ and $\mathrm{H}$ represent the partial molar quantities, and $\mathrm{s}, v$ and $\mathrm{h}$ are extensive properties;

$$
s=\left(\frac{\partial s}{d n}\right)_{P, T} ; v=\left(\frac{\partial V}{d n}\right)_{P, T}
$$

Following that the volume of the adsorbed phase is far less than the bulk volume, i.e. $v_{a} \ll v_{b l k}$, and considering the ideal gas relationship on molar bases, $\mathrm{PV}=\mathrm{RT}$, the above expression can be rearranged as: 
$\left(\frac{d P}{d T}\right)_{C_{a}}=\frac{\Delta s}{v_{b l k}}=\rho_{e q} \Delta s$

Where equilibrium specific density, $\rho_{e q}=\frac{1}{v_{b l k}}=\frac{P}{R T}$

$\frac{1}{P}\left(\frac{d P}{d T}\right)_{C_{a}}=-\frac{\left(s_{a}-s_{b}\right)}{R T}=-\frac{\Delta s_{e}}{R T}$

Recall the internal energy change from the first law of thermodynamics for a reversible closed system (batch process) where differential entropy is given as reduced heat; $d s=\frac{\delta q}{T}$, and at constant temperature and pressure, the differential entropy is related to the specific enthalpy as $\mathrm{d} d s=\frac{\delta h}{T}$. Assuming independence on temperature changes in the process, the above equation can be represented as:

$$
\begin{aligned}
& \frac{1}{P}\left(\frac{d P}{d T}\right)_{C_{a}}=\left(\frac{d \ln P}{d T}\right)_{C_{a}}=-\frac{\Delta s_{a}}{R T}=-\frac{\Delta h_{a}}{R T^{2}}=\frac{q_{i}}{R T^{2}} \\
& -\Delta H=-T \Delta S
\end{aligned}
$$

Where $\Delta \mathrm{s}_{\mathrm{a}}$ and $\Delta \mathrm{h}_{\mathrm{a}}$ represent the sorption entropy and enthalpy respectively, $\mathrm{q}_{\mathrm{i}}$ is the Isosteric heat of sorption of the uptake process. Putting the above equation in van't Hoff form, the isosteric heat becomes

$$
q_{i}=-R\left(\frac{d \ln P}{d\left(\frac{1}{T}\right)}\right)_{C_{a}}>0
$$

The Isosteric heat of adsorption, $q_{i}\left(\mathrm{kJmol}^{-1}\right)$ has been described as the heat of adsorption determined at constant amount of sorbate. As one of the basic requirements for the characterization and optimization of sorption process and as a critical design variable in the process performance estimation, it portrays the specific combined property of sorbent-sorbate combination and gives some indication about the surface energetic heterogeneity.

Truly, the temperature or pressure in liquid medium is outside the supposed ideal gas regime; and density of the bulk phase is not well simplified using the ideal gas expression hence, the more general relationship can apply:

$$
q_{i}=\frac{T}{\rho_{e q}}\left(\frac{d P}{d T}\right)_{C_{a}}
$$

Where $\mathrm{P}$ is the bulk pressure corresponding to the surface excess concentration, hence corresponds to the bulk concentration in liquid medium. The isosteric heat is the presumed heat of sorption defined by the above 
isosteric heat expressions. In sorption, from engineering viewpoint, the absolute adsorbed amount is not practically obtainable via the widely applied volumetric method, "batch system" though it can be approximated numerically hence the isosteric heat can best be apparent.

The isosteric heat of sorption can be estimate from the mobile and localized sorption enthalpy, where the localized sorption enthalpy represent the isoexcess heat, the mobile sorption enthalpy describe the heat involvement at the immediate vicinity of the adsorbed layer cognitive of the experimentally indeterminate fraction of the isosteric heat. The theoretical basis of this estimation is revolved around the temperature-kinetic relationship in fluids. It follows that during sorption, mobility of the sorptive into and within the adsorbed phase involves kinetic energies: translational, rotational and vibrational that is normal to the surface, all expressed as RT. At the vicinity of adsorbed layer, kinetic energy is mainly vibrational and the enthalpy of sorption $\left(\mathrm{H}_{\mathrm{sorb}}\right)$ involves the binding energy $\left(\mathrm{E}_{0}\right)$ and the sorptive excitement (translational, rotational and/or vibrational). The enthalpy of sorptive in the bulk, far and near the adsorbed surface vicinity is given as $\mathrm{H}_{\text {blk }}=5 / 2 \mathrm{RT}$. On the basis of a unit sorptive, for mobile sorption, the total excitement is $2 \mathrm{RT}$ and for localized sorption, 3RT hence the enthalpy: $\mathrm{H}_{\text {sorb }}$ $=-\mathrm{E}_{0}+2 \mathrm{RT}$ and $\mathrm{H}_{\text {sorb }}=-\mathrm{E}_{0}+3 \mathrm{RT}$ respectively giving the mobile $\left(q_{\text {isom }}\right)$ and localized $\left(q_{\text {isoL }}\right)$ isosteric heats as $q_{\text {isom }}=\mathrm{E}_{0}+1 / 2 \mathrm{RT}$ and $q_{\text {isoL }}=\mathrm{E}_{0}-1 / 2 \mathrm{RT}$ respectively.

Generally, the isosteric heat is given as:

$q_{i s o}=a+(N-1) \delta$

Where $N=$ number of atoms in molecule, $a=\mathrm{E}_{0}+1 / 2 \mathrm{RT} ; \delta=-1 / 2 \mathrm{RT}$ for mobile phase sorption and $a=\mathrm{E}_{0}-1 / 2 \mathrm{RT} ; \delta=2 \mathrm{RT}$ for the localized phase

\section{Isoexcess Heat}

Considering the thermodynamics of sorption in liquid medium, the surface excess layer, the vicinity within the thin film surrounding a sorbent surface, is seen as a distinct phase, with a thermodynamic state that is completely characterized by an area A, a spreading sorptive density, and a surface excess concentration, $C_{a}=\frac{q_{e}}{A}$.

The isosteric heat is the heat of absolute sorption which is referred to the total number of sorptive molecules that are located within the adsorbed phase/volume. However, adsorbed volume cannot be measured directly since the density profile of the adsorbed phase is not experimentally known. The reason being that at experimental time when adsorption is zero, the finite volume of the adsorbed phase would have contained an amount of the 
adsorbed species (sorbate) given by the bulk density. Therefore, this amount is often excluded from the experimentally measured uptake where density at time, $\mathrm{t}$ is subtracted from the initial density at time, zero; when there was no sorption on the surface. This subtraction gives the excess adsorbed amount which is the amount of sorptive present in the adsorbed layer in excess of the bulk density. It is typical therefore to report the isoexcess heat of sorption since it is standard practice to report sorption as a Gibbs surface excess quantity. Hence, excess quantities can be substituted for the absolute quantities in the above expressions:

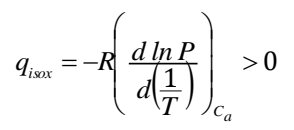

At Non-ideality;

$$
q_{\text {isx }}=\frac{T}{\rho_{e q}}\left(\frac{d P}{d T}\right)_{C_{a}}
$$

Since bulk pressure, $\mathrm{P}$ is corresponding to the surface concentration, hence to the bulk concentration in liquid medium;

$$
\begin{aligned}
& P=C_{t} R T \\
& q_{\text {iox }}=-R\left(\frac{d \ln C_{t}}{d\left(\frac{1}{T}\right)}\right)_{C_{a}} \text { and } q_{\text {iox }}=\frac{R T^{2}}{C_{e q}}\left(\frac{d C_{t}}{d T}\right)_{C_{a}}
\end{aligned}
$$

Unless the adsorbed state is characterized by a very large additional entropy (perhaps from vibrations), it is presupposed that adsorption is always exothermic $\left(\Delta \mathrm{H}_{\mathrm{a}}<0\right)$. As applicable in different batch systems, integrating the above excess heat expression in equilibrium at the corresponding system

temperature would give van't Hoff plot of $\ln \mathrm{C}_{\mathrm{t}}$ and $\mathrm{T}^{-1}$ with slope $\frac{q_{\text {isox }}}{R}$. These excess heat expressions are well applicable in liquid medium being that experimentally, the initial concentration is fixed (hence controllable) and the amount adsorbed measurable, but it is very difficult to fix the adsorbed amount to measure the equilibrium concentration. Based on the principles or assumptions of ideality on which the above excess heat expression was formulate, liquids, like all gases tend toward ideality where interactions are improbable and the total volume of the system is approximately unchanged by including the molecules in it.

Materials and Methods

Materials 
All the chemicals used were analytical grade reagents in the Springboard Laboratory, Udoka Housing Estate, Awka, Anambra state.

The Ekowe clay was mined from Federal Polytechnic Ekowe, Bayelsa state, wash with water from the site to remove sand and suspended organic matter, then sun-dried in a lining covered Petri dish-like-basin. The dried clay was purified by steeping in distilled water for about 12 hours to dissolve chemical elements from the source water, then washed trice. The purified clay was sun-dried under a lining cover for four hours then dried in the oven at $45^{\circ} \mathrm{C}$ for 24 hours and cooled in a desiccator for 5hours.

The dried clay was thermally and chemically activated before used for the adsorption of Methylene blue dye from its aqueous solution. Half of the dried clay was heated at $750^{\circ} \mathrm{C}$ for 4 hours, cooled, crushed and sieved with $45 \mu \mathrm{m}$ sieve (200 Mesh ASTM) to produce the Natural Ekowe Clay (NEC). The second half was mixed with sufficient amount of $1.6 \mathrm{M} \mathrm{H}_{2} \mathrm{SO}_{4}$ (aq) solution and heated in reflux in an electronic thermostatic water tank for $3 \mathrm{hrs}$ at $90^{\circ} \mathrm{C}$. After cooling, the activated clay was washed severally with distilled water until neutral $\mathrm{pH}$ was attained then dried at $40^{\circ} \mathrm{C}$ for about $6 \mathrm{hrs}$. The dried clay was heated at $750^{\circ} \mathrm{C}$ for $4 \mathrm{hrs}$ then cooled, crushed and sieved with $45 \mu \mathrm{m}$ sieve (200 Mesh ASTM) to produce the Activated Ekowe Clay (AEC).

The Basic dye, Methylene Blue (MB) $\left(\mathrm{C}_{16} \mathrm{H}_{18} \mathrm{~N}_{3} \mathrm{SCl} .3 \mathrm{H}_{2} \mathrm{O}\right)$ was purchased at Bridge-head market Onitsha, Anambra state and used without any modification. $0.1 \mathrm{~g}$ of the dye was dissolved in $100 \mathrm{~mL}$ distilled water to produce $1 \mathrm{~g} / \mathrm{L}$ stock solution. The stock solution was diluted to produce the experimental dye solution.

\section{Methods}

The kinetic runs were carried out at times 50, 80 and 110min for temperatures 25,30 and $40^{\circ} \mathrm{C}$. with $50 \mathrm{~mL}$ dye solution and $0.4 \mathrm{~g}$ clay. For each run, $0.4 \mathrm{~g}$ clay was added to $50 \mathrm{~mL}$ dye solution and the mixture was agitated at the desired temperature in TIPO GTR90 thermostatic stirrer water bath for the given time interval. At the elapse of time, the mixture was centrifuged $(\sim 15000 \mathrm{rpm})$ for $15 \mathrm{~min}$. and the supernatant solutions were decanted for the determination of the absorbance using the APEL PD$3000 \mathrm{UV}$ spectrophotometer at a wavelength of $610 \mathrm{~nm}$.Absorbance was converted to concentration using the linear calibration graph.

Uptake, the amount adsorbed, $(\mathrm{m} g / \mathrm{g})$ was calculated using the mass balance equation:

$$
q_{t}=\frac{\left(C_{o}-C_{t}\right) V}{m}=\frac{V C_{a}}{m}
$$


Where $q$ is amount adsorbed $(\mathrm{m} g / g), C_{o} / C_{e}$ is initial/equilibrium dye concentration $(\mathrm{mg} / L), V$ is volume of dye solution $(L)$, and $m$ is dry weight of clay $(g)$.

\section{Error Analysis}

The fitness of kinetic models were tested with statistical tools; Correlation coefficient (R), Coefficient of Determination $\left(\mathrm{R}^{2}\right)$, Standard Error $\left(\chi^{2}\right)$, and Normalized standard deviation $(\Delta \mathrm{q})$ using Microsoft Excel. These standard and error estimation were considered on the experimental $\left(q_{e, e x p}\right)$ and calculated $\left(q_{e, c a l}\right)$ equilibrium uptakes uptake for the number of experimental runs (n) used.

I. Standard error of estimate (Spiegel and Stephens 2008)

$$
\chi^{2}=\left(\frac{\sum\left(q_{\infty, e x p}-q_{\infty, c a l}\right)^{2}}{n}\right)^{0.5}
$$

II. The deviation of the calculated uptake from the experimentally observed can be estimated by the Normalized standard deviation (Sölener, et al, 2008; Nagashanmugam and Srinivasan, 2011) or the Hybrid fractional error (Gulipalli et al, 2011) given respectively:

$$
\Delta q=100\left(\frac{\sum\left(q_{\infty, e x p}-q_{\infty x a l}\right)^{2}}{q_{\infty, x \times p}}\right)^{0.5}
$$

\section{Results and Discussion}

The negative slope of the $\ln (\mathrm{K} / \mathrm{T})$ vs $(1 / \mathrm{T})$ plot by the Tolman's interpretation implied that $\Delta \mathrm{H}^{*}$, the energy difference between the sorbate and the sorptive, is equal to the average energy of the dye molecules that are adsorbed minus the average energy of all the dye molecules in the aqueous solution (Tolman, 1927).

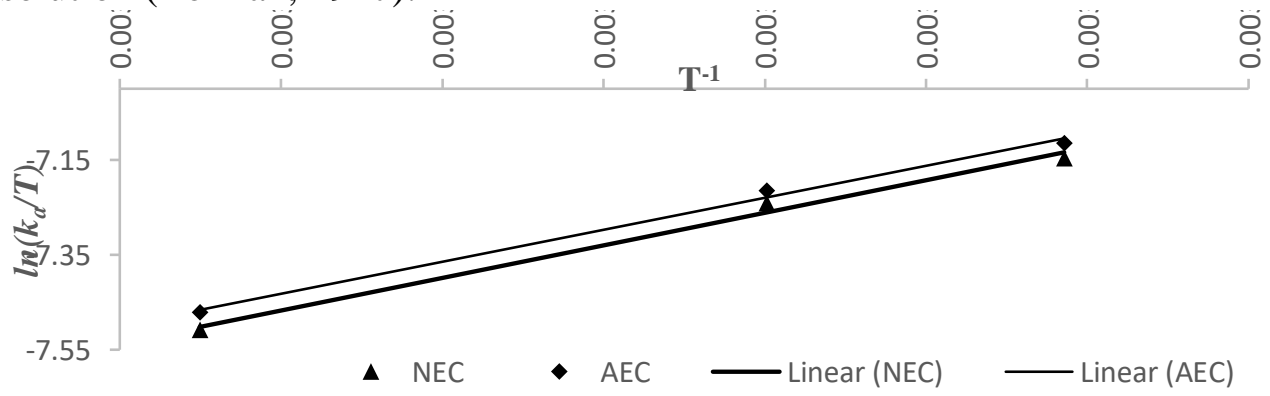

Figure 1: Activation thermodynamics plot - the Eyring plot (straight line): $\ln \left(k_{a} / T\right)$ vs $T^{-1}$

From tabs. 1 and2, the value of $\mathbb{E}$ lies between 2 and $29 \mathrm{~kJ} / \mathrm{mol}$, indicating that the bond of uptake was only due to van der Waals interactions characteristic of physisorption. Positive activation energy, $\mathbb{E}$ which defines 
the endothermicity of the adsorption onset have always been reported for dye stuff (saha and sch... 2010). The positive $(\mathbb{E})$ indicated the involvement of external energy (potential) which was evident with the application of mechanical agitation (work done) in the clay-dye solution mixture. Conventionally, $\mathbb{E}$ increases with temperature and as shown in Tabs 1 and 2, implies the slow and decreased sorption with increase in temperature.

The activation enthalpy, $\Delta \mathrm{H}^{*}$ for Methylene blue uptake onto modified Ekowe clay depict less negative value hence indicated that the uptake proceeded at a fast rate compliant to the significant values of $\mathrm{k}_{2}$ which decreases with increasing temperature. This confirms the exothermic nature of the surface interaction spontaneously favoured by decrease in temperature. In the exothermic MB uptake; characterized by negative $\Delta \mathrm{H}^{*}$, energy absorbed in dye solvation bond breaking is less than the total energy released in clay-dye and clay solvation bond making, resulting in the release of extra energy in the form of heat. However, $\mathbb{E}$ increased with increase in temperatuure supporting the slow in sorption with increase in temperature.

Table 1: Thermodynamics activation parameter for Natural Ekowe Clay (NEC): equilibrium uptake, $\mathrm{q}_{\mathrm{e}}(\mathrm{mg} / \mathrm{g})$; Standard error, $\mathcal{X}^{2}$; Normalized standard deviation, $\Delta \mathbf{q}(\%)$; Second order rate constant, $\mathrm{k}_{2}$ (g/mg.s); Activation energy, $\mathbb{E}(\mathrm{kJ} / \mathrm{mol})$; Activation enthalpy, $\Delta \mathrm{H}^{*}(\mathrm{~kJ} / \mathrm{mol})$; entropy, $\Delta \mathrm{S}^{*}(\mathrm{~kJ} / \mathrm{mol} . \mathrm{K})$; and free energy of Activation, $\Delta \mathrm{G}^{*}(\mathrm{~kJ} / \mathrm{mol})$.

\begin{tabular}{cccccccccc}
\hline $\mathrm{T}(\mathrm{K})$ & $\mathrm{q}_{\mathrm{e} e x p}$ & $\mathrm{q}_{\mathrm{e}, \text { cal }}$ & $\mathcal{X}^{2}$ & $\Delta \mathrm{q}$ & $\mathrm{k}_{2}$ & $\mathbb{E}$ & $\Delta \mathrm{H}^{*}$ & $\Delta \mathrm{S}^{*}$ & $\Delta \mathrm{G}^{*}$ \\
\hline 298 & 1.331 & 1.367 & & & 0.235 & 2.21 & -0.28 & -321 & 95.29 \\
303 & 1.306 & 1.344 & 0.123 & \multirow{2}{*}{6.83} & 0.217 & 2.25 & -0.28 & -321 & 96.90 \\
313 & 1.212 & 1.261 & & & 0.172 & 2.33 & -0.28 & -321 & 100.1 \\
\hline
\end{tabular}

Table 2: Thermodynamics activation parameter for Activated Ekowe Clay (AEC): equilibrium uptake, $\mathrm{q}_{\mathrm{e}}(\mathrm{mg} / \mathrm{g})$; Standard error, $\chi^{2}$; Normalized standard deviation, $\Delta \mathbf{q}(\%)$; Second order rate constant, $\mathrm{k}_{2}$ (g/mg.s); Activation energy, $\mathbb{E}(\mathrm{kJ} / \mathrm{mol})$; Activation enthalpy,

$\Delta \mathrm{H}^{*}(\mathrm{~kJ} / \mathrm{mol})$; entropy, $\Delta \mathrm{S}^{*}(\mathrm{~kJ} / \mathrm{mol} . \mathrm{K})$; and free energy of Activation, $\Delta \mathrm{G}^{*}(\mathrm{~kJ} / \mathrm{mol})$.

\begin{tabular}{cccccccccc}
\hline $\mathrm{T}(\mathrm{K})$ & $\mathrm{q}_{\mathrm{e} e x p}$ & $\mathrm{q}_{\mathrm{e}, \mathrm{cal}}$ & $\mathcal{X}^{2}$ & $\Delta \mathrm{q}$ & $\mathrm{k}_{2}$ & $\mathbb{E}$ & $\Delta \mathrm{H}^{*}$ & $\Delta \mathrm{S}^{*}$ & $\Delta \mathrm{G}^{*}$ \\
\hline 298 & 1.468 & 1.503 & & & 0.242 & 2.20 & & & 94.88 \\
303 & 1.462 & 1.499 & 0.118 & 5.85 & 0.223 & 2.24 & -0.27 & -319 & 96.48 \\
313 & 1.368 & 1.414 & & & 0.178 & 2.33 & & & 99.67 \\
\hline
\end{tabular}

Where $\mathbf{q}_{\mathbf{e}} \exp$ is the experimental equilibrium uptake $(\mathrm{mg} / \mathrm{g})$ and $\mathbf{q}_{\mathbf{e}, \mathbf{c a l}}$ is the graphical estimated equilibrium uptake $(\mathrm{mg} / \mathrm{g})$. Following the use of the second order model in estimating the equilibrium kinetic data, the very low standard error suggested that second-order kinetic best describe the MB uptake. The values of the normalized standard deviation $\Delta \mathrm{q}$ showed very low deviations $(<10 \%)$ validating the experimental uptake data (Emeniru et al. 2015).

The larger the negative value of $\Delta \mathrm{S}^{*}$ the slower the adsorption, therefore, the less negative value of $\Delta \mathrm{S}^{*}$, the difference in entropy between 
the sorbate and the sorptive, as shown in Tabs 2 and 3 depicted that though the dye molecules at the adsorbed phase lost some translational entropy to possess a more ordered structure than in the bulk, they were not frozen on the clay surface rather the transitional, rotational and vibrational degree of freedom of the dye sorptive at the clay surface remained, barely; this is favoured at low temperature and accounts for possible desorption thus illustrated the uptake to be physisorption. The negative activation entropy also explained an associative uptake that left the sorbent structure with no significant change, an attribute of physisorption.

The positive $\Delta \mathrm{G}^{*}$ values are indicative that free energy generated seemingly from physical work in the adsorption process - stirring and/or vibration, is required to initiate the mobility of $\mathrm{MB}$ molecules hence the activation of the uptake.

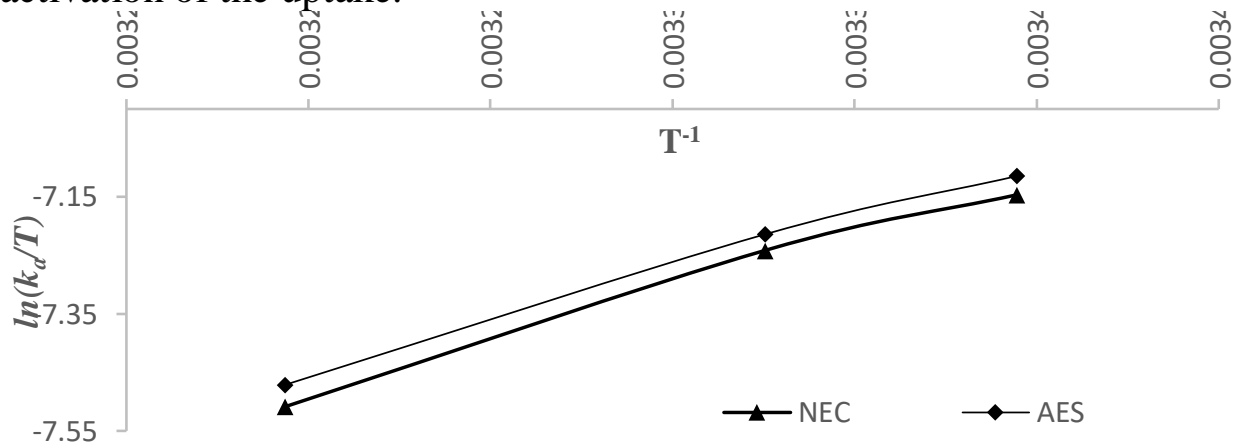

Figure 2: Activation thermodynamics plot - the Eyring plot (concave curve): $\ln \left(k_{a} / T\right) v s T^{l}$

In the broad field of kinetics, nonlinear Eyring plot can be observed as illustrated in Fig 2 above, and they are almost always concave which can be attributed to several factors, the most common interpretation being an indication that at least two different rate-limiting steps (film and intraparticle diffusion) are involved in the sorption complimentarily.

Isosteric heat otherwise referred as iso-heat has been tagged the most relevant thermodynamic variable to describe the heat effects during sorption process (Saha and Chowdhury, 2010). Its magnitude has always implied the sorption mechanism driving the process: chemisorption $(80-400 \mathrm{~kJ} / \mathrm{mol})$ or physisorption $(<80 \mathrm{~kJ} / \mathrm{mol}$ ) (Saha and Chowdhury, 2010). The Localized isoheat, $q_{i s o L}$ as shown in Tab. 3 and $\mathbf{4}$ is low relative to the value of the Mobile iso-heat conforming to the postulate of progressive decreasing sorption potential and increasing iso-heat accompanied by further decrease in sorption potential and fall in the iso-heat as the sorption tends to equilibrium when multilayer uptake is very likely, heterogeneously. 
Table 3: Thermodynamics heat of adsorption - Natural Ekowe Clay (NEC): Isoexcess heat, $\mathrm{q}_{\text {isox }}$; Localized isosteric heat, $\mathrm{q}_{\text {iso }}$; Mobile Iso-heat, $\mathrm{q}_{\text {iso }}$; thermodynamic enthalpy, $\Delta \mathrm{H}$; and thermodynamic entropy, $\Delta \mathrm{S}$.

\begin{tabular}{ccccccc}
\hline $\mathrm{T}(\mathrm{K})$ & $q_{\text {isox }}$ & $q_{\text {isoM }}$ & $q_{\text {isoL }}$ & $\Delta \mathrm{H}$ & $\Delta \mathrm{S}$ & $\Delta \mathrm{G}$ \\
\hline 298 & & 3.441 & 0.964 & & -8.94 & -739 \\
303 & 2.665 & 3.504 & 0.984 & -2.665 & -8.79 & -835 \\
313 & & 3.628 & 1.026 & & -8.51 & -1186 \\
\hline
\end{tabular}

Table 3: Thermodynamics heat of adsorption - Activated Ekowe Clay (AEC): Isoexcess heat, $\mathrm{q}_{\text {isox }}$; Localized isosteric heat, $\mathrm{q}_{\text {isoL }}$; Mobile Iso-heat, $\mathrm{q}_{\text {iso }}$; thermodynamic enthalpy, $\Delta \mathrm{H}$; and thermodynamic entropy, $\Delta \mathrm{S}$.

\begin{tabular}{ccccccc}
\hline $\mathrm{T}(\mathrm{K})$ & $q_{\text {isox }}$ & $q_{\text {isoM }}$ & $q_{\text {isoL }}$ & $\Delta \mathrm{H}$ & $\Delta \mathrm{S}$ & $\Delta \mathrm{G}$ \\
\hline 298 & & 3.446 & 0.969 & & -8.28 & -299 \\
303 & 2.467 & 3.509 & 0.989 & -2.467 & -8.14 & -327 \\
313 & & 3.633 & 1.031 & & -7.88 & -653 \\
\hline
\end{tabular}

The increase in the isosteric heats, both the mobile and localized phase, further authenticate the exothermicity of the physisorption and its favourability at low temperature. This is shown in the increased isosteric heat as recorded in Tabs 3 and 4 indicating low uptake said to be characteristic of high isosteric heat.

The Gibbs energy change, $\Delta \mathrm{G}$ indicated that the dye molecules at the adsorbed phase has less free energy compared to the dye sorptive in the bulk solution hence the adsorption of the dye onto the clay surface can occur without the influence of external energy, that is, spontaneously. The increased negativity of $\Delta \mathrm{G}$ with temperature depict the increased loss of free energy by the dye sorbate upon sorption on the clay surface which explains why the intermolecular attraction force typical of physisorption reduces with increasing temperature hence supporting that the dye uptake is exothermic. The higher negativity of $\Delta \mathrm{G}$ in the NEC relative to AEC infers that physisorption is stronger in AEC than NEC, therefore AEC has higher uptake capability and adsorbed the MB dye better than NEC. The high negative $\Delta \mathrm{G}$ with increased temperature indicated longer time for equilibrium due to desorptive interruptions characteristic of the high system energy.

\section{Conclusion}

The second order MB uptake is expressed by the close values of $\mathbf{q}_{\mathbf{e} \text { exp }}$ and $\mathbf{q}_{\mathbf{e}, \mathbf{c a l}}$, the low standard error and deviation, and the Eyring concave plot suggesting more than one sorption kinetic mechanism for the transfer of the dye molecules.

The positive values of $\Delta \mathrm{G}^{*}$ corresponding to the positive activation energy $(\mathbb{E})$, explained the introduction of external potential, but not heat, that activated the MB molecules presumptively, mechanistic induced potentials 
from stirring and/or agitation. Characterized by less negative value of $\Delta \mathrm{S}^{*}$, MB uptake was fast and associative with the MB molecules at the clay surface losing translational entropy to possess a better physisorptive ordered structure on the surface intact. The connection of $\Delta \mathrm{H}$ and $\Delta \mathrm{H}^{*}$ defined the exothermicity of the process and its activation; revealing that the release of heat in the system is as a result of the negative energy difference (energy release) between molecular attraction on clay and the dissociation of MB from the solution.

By the realities of the adsorption experiment, $\Delta \mathrm{H}$ is the approximate of the isoexcess heat of adsorption $\left(\mathrm{q}_{\text {isox }}\right)$. The increase in the isosteric heats, both the mobile and localized phase, with temperature, authenticate the exothermicity of the physisorption. The negative $\Delta \mathrm{G}$ express the spontaneity of the MB uptake at all considered temperature, though, increased negativity with temperature points to longer time for uptake equilibrium. The higher negativity of $\triangle \mathrm{G}$ in the NEC relative to AEC infers that physisorption is stronger in AEC than NEC, therefore AEC has higher uptake capability and adsorbed the MB dye better than NEC. The high negative $\triangle \mathrm{G}$ with increased temperature indicated.

\section{References:}

1. Aharoni, C. \& Sparks, D. L. (1991). Kinetics of Soil Chemical Reactions - A Theoretical Treatment, in D. L. Sparks and D. L. Suarez (Eds.), Rates of Soil Chemical Processes, Soil Science Society of America, Madison, WI, 1-18.

2. Aksakal, O. \& Ucun, H. (2010). Equilibrium, kinetic and thermodynamic studies of the biosorption of textile dye (Reactive Red 195) onto Pinus sylvestris L. Journal of Hazardous Materials, 181, 666-672. ISSN: 0304-3894.

3. Alkan, M., Dogan, M., Turhan, Y., Demribas, O. \& Turan, P. (2008). Adsorption kinetics and mechanism of maxilon blue $5 \mathrm{G}$ dye on sepiolite from aqueous solutions. Chemical Engineering Journal, 139, 213-223.

4. Chowdhury, S., Mishra, R., Saha, P. \& Kushwaha, P. (2010). Adsorption thermodynamics, kinetics and isosteric heat of adsorption of malachite green onto chemically modified rice husk. Desalination, doi:10.1016/j.desal.2010.07.047.

5. Chowdhury, S. \& Saha, P. (2010). Sea shell powder as a new adsorbent to remove Basic Green 4 (Malachite Green) from aqueous solutions: Equilibrium, kinetic and thermodynamic studies. Chemical Engineering Journal, doi:10.1016/j.cej.2010.08.050.

6. Emeniru, D. C., Onukwuli, O. D., Wodu, D. P., \& Okoro, B. I. (2015). The Equilibrium and Thermodynamics of Methylene Blue 
Uptake onto Ekowe Clay; Influence of Acid Activation and Calcination. International Journal of Engineering and Applied $\begin{array}{lllll}\text { Sciences } & \text { (IJEAS), } & 2 & \text { (5), } & \text { 17-25. }\end{array}$ https://www.ijeas.org/download_data/IJEAS0205015.pdf

7. Han, R., Zhang, L., Song, C., Zhang, M., Zhu, H. \& Zhang, L. (2010). Characterization of modified wheat straw, kinetic and equilibrium study about copper ion and methylene blue adsorption in batch mode. Carbohydrate Polymers. 79, 1140-1149. ISSN: 0144-8617.

8. Klaus Christmann, (2011). Energetics of Adsorption and Desorption. Introductory Block Course "Chemistry and Physics of Surfaces"

9. Klaus Christmann (2012). Thermodynamics and Kinetics of Adsorption. IMPRS- Lecture Series 2012: 'Experimental and Theoretical Methods in Surface Science'.

10. Levine R. D. (1979). Free energy of activation. Definition, properties, and dependent variables with special reference to "Linear" free energy relations. Journal of physical chemistry, 83 (1), $159-169$.

11. Myers, A. L. (2004). Thermodynamics of adsorption. Chemical thermodynamics for industry, 243-252.

12. Ölenera, M., Tunalib, S., Özcanc, A. S., Özcanc, A. \& Gedikbey, T. (2008). Adsorption characteristics of Lead (II) ions onto the clay/poly(methoxyethyl)acrylamide (PMEA) composite from aqueous solutions. Desalination 223, 308-322.

13. Sarici-Ozdemir, C. (2012). Adsorption and desorption kinetics behaviour of Methylene Blue onto activated carbon. Physicochemical Problems of Mineral Processing, 48(2), 441454.www.minproc.pwr.wroc.pl/journal/

14. Smith, J. M., Van Ness H. C. \& Abbot, M. M. Introduction to Chemical Engineering Thermodynamics, Fifth Edition. McGrawHill(1996). ISNB 10.007059239X

15. Smith F. C. and Van Ness. Introduction to Chemical Engineering Thermodynamics

16. Sparks, D.L \& Jardine, P.M. (1984). Comparison of kinetic equations to describe Potassium-calcium exchange in pure and in mixed system. Soil Science, $138 \quad$ (2), 115-122.

17. Tolman, R. C. (1927).Statistical Mechanics with Applications to Physics and Chemistry (Chemical Catalog Co., New York), pp. 260270. 